\title{
Visualizing the Dynamics of Dystrophin Protein Complex Assembly in Living Cells
}

\author{
R. A. Draviam, ${ }^{*}$ B. Wang, ${ }^{* *}$ S. Shand, ${ }^{*}$ X. Xiao, ${ }^{* *}$ S. C. Watkins* \\ *Department of cell biology and physiology, University of Pittsburgh School of Medicine, \\ Pittsburgh, PA 15261 \\ **Department of molecular genetics and biochemistry, University of Pittsburgh School of Medicine, \\ Pittsburgh, PA 15261
}

The Duchenne and Limb girdle muscular dystrophies (DMD, LGMD) are a heterogeneous group of genetic disorders that affects about 1 in 3500 males each year. Primary mutations in the dystrophin gene result in the absence of the protein in DMD, and mutations in any one of four sarcoglycan $(\alpha, \beta$, $\Delta, \gamma)$ genes results in a loss of the entire sarcoglycan complex in LGMD [1] (Fig. 1). In humans these myopathies result in progressive muscle weakness and eventually patient death due to cardiac failure. Despite selective in vitro studies examining the assembly pathway of the dystrophin and sarcoglycan complex in fixed systems, the precise mechanism of how these genes behave in living cells remained unknown. In the present study we have developed and implemented a model system to study dystrophin protein complex (DPC) assembly in living cells. We demonstrate that dystrophin associated proteins (DAP) follow a sequential assembly process which can be observed in real time.

Here, we report the construction and characterization of a $4.5 \mathrm{~kb}$ minidystrophin-EGFP fusion gene, and a $1.9 \mathrm{~kb} \alpha$-sarcoglycan-EGFP fusion gene. Live cell microscopy is utilized to show that minidystrophin is deposited at the plasma membrane (PM) (Fig. 2B-E) soon after expression in living muscle cells, early in differentiation. Once at the surface we demonstrate that minidystrophin is firmly anchored to the sarcolemma via interactions with select DPC members that precede the expression of dystrophin and the sarcoglycans (Fig. 2A). At the same time point early during differentiation, $\alpha$-sarcoglycan-EGFP does not properly assemble at the PM in living cells (Fig. 3A). DiI lipid dyes and multi-color live cell imaging is used to show that $\alpha$-sarcoglycan is confined to motile vesicular structures (Fig. 3B-I) that accumulate in large clusters approximately 5 hours after expression, resulting in cell death. In mature mouse muscle containing all normal sarcoglycans, confocal microscopy is used to demonstrate that $\alpha$-sarcoglycan-EGFP correctly localizes to the sarcolemma of the muscle fiber.

Together these data support a model whereby dystrophin expression and localization at the PM precedes and is independent of the sarcoglycans. It is not until later during myotube differentiation that the sarcoglycan proteins assemble and cooperate in a specific fashion to translocate to the PM. These results are the first to demonstrate the systematic and stepwise assembly of the dystrophin protein complex in living cells. Understanding the basic molecular mechanisms that underlie DPC assembly is crucial for the proper development of gene therapies for the muscular dystrophies in humans.

References

[1] Draviam, R., L. Billington, et al. (2001). Muscle Nerve 24(2): 262-72.

[2] This work is supported by a grant from the National institute of health, under contract \#U54AR050733. 


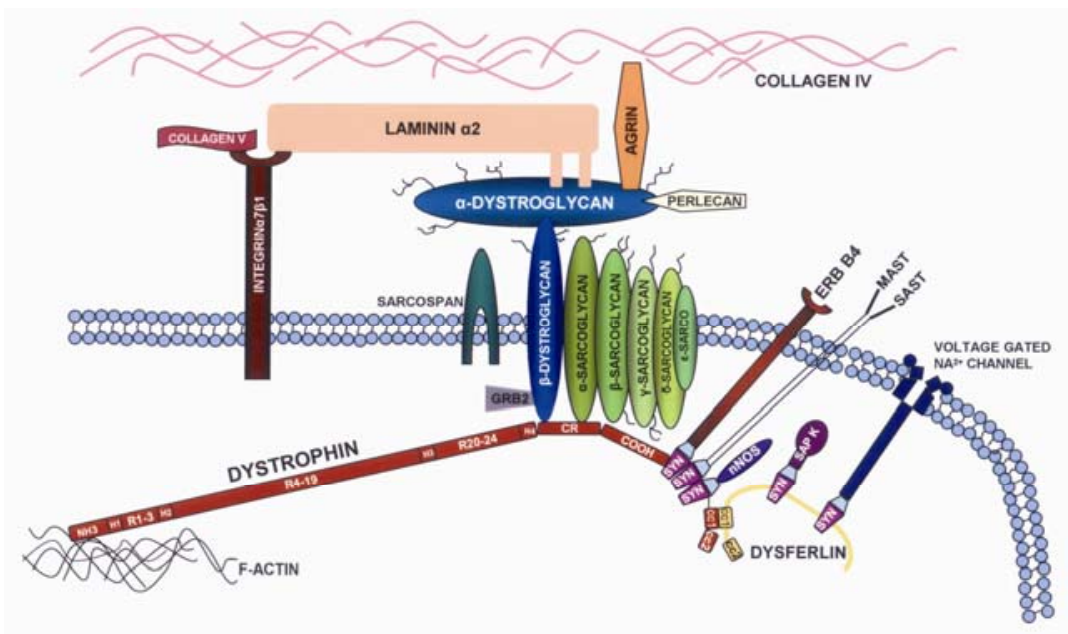

Figure 1- Schematic of Dystrophin Protein Complex in a skeletal muscle cell.
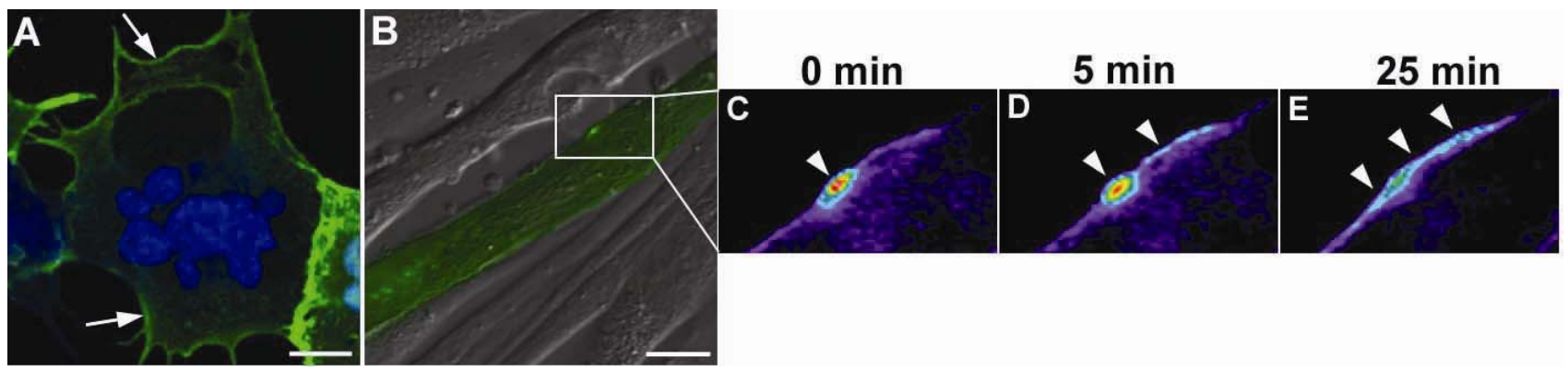

Figure 2- Minidystrophin-EGFP assembly at the plasma membrane of living cells. Panel A shows Minidystrophin-EGFP (green) at the plasma membrane of non muscle cells (arrow), lacking sarcoglycans, by confocal microscopy (nuclei in blue). Panels B-E shows the fusion protein being distributed at the surface (arrowhead) of a living muscle myotube in culture. Bar= 15um.

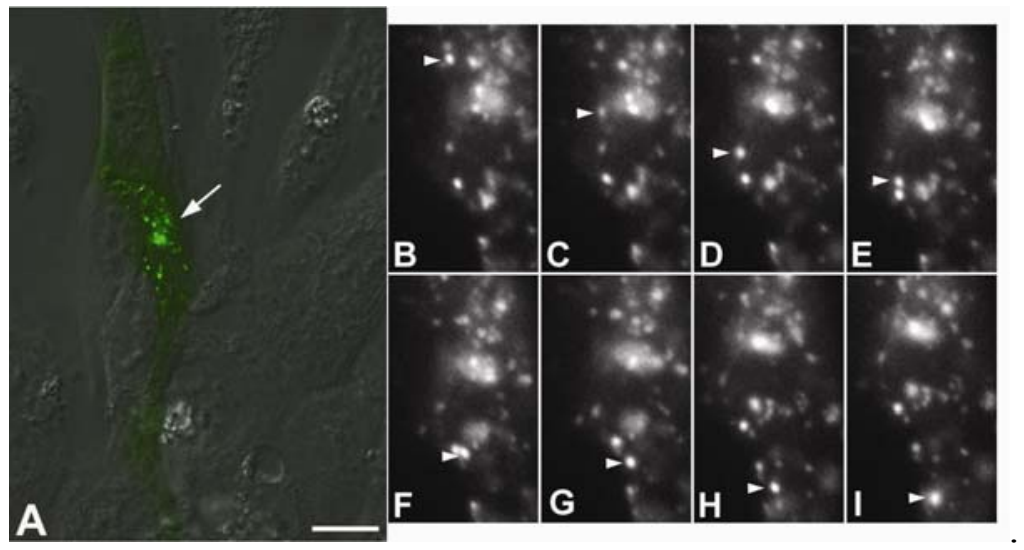

Figure 3- $\alpha$-sarcoglycan-EGFP in living muscle cells early in differentiation. A-sarcoglycan (green) accumulates in intercellular vesicular structures (arrow), without the other sarcoglycans. Panels B-I show the vesicular motion of $\alpha$-sarcoglycan (arrowhead), without proper plasma membrane deposition. Bar= $15 \mathrm{um}$. 